\title{
Hippocampal function in schizophrenia and bipolar disorder
}

\author{
J. Hall $^{1 *}$, H. C. Whalley ${ }^{1}$, K. Marwick ${ }^{1}$, J. McKirdy ${ }^{1}$, J. Sussmann ${ }^{1}$, L. Romaniuk ${ }^{1}$, E. C. Johnstone ${ }^{1}$, \\ H. I. Wan ${ }^{2}$, A. M. McIntosh ${ }^{1}$ and S. M. Lawrie ${ }^{1}$ \\ ${ }^{1}$ Division of Psychiatry, University of Edinburgh, Royal Edinburgh Hospital, Edinburgh, UK \\ ${ }^{2}$ Clinical Translational Medicine, Wyeth Research, Collegeville, PA, USA
}

Background. The hippocampus plays a central role in memory formation. There is considerable evidence of abnormalities in hippocampal structure and function in schizophrenia, which may differentiate it from bipolar disorder. However, no previous studies have compared hippocampal activation in schizophrenia and bipolar disorder directly.

Method. Fifteen patients with schizophrenia, 14 patients with bipolar disorder and 14 healthy comparison subjects took part in the study. Subjects performed a face-name pair memory task during functional magnetic resonance imaging (fMRI). Differences in blood oxygen level-dependent (BOLD) activity were determined during encoding and retrieval of the face-name pairs.

Results. The patient groups showed significant differences in hippocampal and prefrontal cortex (PFC) activation during face-name pair learning. During encoding, patients with schizophrenia showed decreased anterior hippocampal activation relative to subjects with bipolar disorder, whereas patients with bipolar disorder showed decreased dorsal PFC activation relative to patients with schizophrenia. During retrieval, patients with schizophrenia showed greater activation of the dorsal PFC than patients with bipolar disorder. Patients with schizophrenia also differed from healthy control subjects in the activation of several brain regions, showing impaired superior temporal cortex activation during encoding and greater dorsal PFC activation during retrieval. These effects were evident despite matched task performance.

Conclusions. Patients with schizophrenia showed deficits in hippocampal activation during a memory task relative to patients with bipolar disorder. The disorders were further distinguished by differences in PFC activation. The results demonstrate that these disorders can distinguished at a group level using non-invasive neuroimaging.

Received 29 January 2009; Revised 1 July 2009; Accepted 7 July 2009

Key words: Bipolar disorder, fMRI, hippocampus, prefrontal cortex, schizophrenia.

\section{Introduction}

Schizophrenia and bipolar disorder are common, disabling psychiatric illnesses that together affect one in 50 people at some stage in their lifetime. There are no diagnostic tests or specific symptoms that uniquely distinguish the two disorders, which are instead separated on the basis of characteristic clusters of symptoms and their longitudinal course. Evidence from imaging studies of each disorder suggests that there may be differences between schizophrenia and bipolar disorder in the structure and function of the hippocampal formation, a region of the brain known to play a prominent role in memory formation

\footnotetext{
* Address for correspondence: Dr J. Hall, Division of Psychiatry, University of Edinburgh, Kennedy Tower, Royal Edinburgh Hospital, Edinburgh EH10 5HF, UK.

(Email : jhall5@staffmail.ed.ac.uk)
}

(Heckers, 2001; Frey et al. 2007). However, to date no studies have directly compared hippocampal function in schizophrenia and bipolar disorder patients using non-invasive neuroimaging techniques.

There is extensive evidence of abnormalities in both the structure and the function of the hippocampus in schizophrenia (Heckers, 2001). Structural neuroimaging studies have shown reduced hippocampal volumes in the disorder, a finding that has been confirmed by meta-analysis (Wright et al. 2000). Hippocampal volume reductions are also seen in the unaffected and at-risk relatives of individuals with schizophrenia, suggesting that such changes are in part heritable and are not simply secondary to the consequences of illness (Boos et al. 2007). Behavioural studies have shown memory deficits in schizophrenia in tasks known to depend on the hippocampus, such as tests of episodic and associative memory (Aleman et al. 1999; Danion et al. 1999; Achim \& Lepage, 2003; 
Toulopoulou et al. 2003). Functional neuroimaging studies have confirmed decreased recruitment of the hippocampus in subjects with schizophrenia, which is particularly pronounced in hippocampal memory tasks (Heckers et al. 1998; Heckers, 2001; Achim \& Lepage, 2005; Ongur et al. 2006).

Studies of hippocampal structure and function in bipolar disorder have in general revealed less evidence for hippocampal pathology. Structural imaging studies of bipolar disorder have failed to find consistent evidence of alterations in hippocampal volume, although there is some evidence of volume reduction in paediatric onset cases (Haldane \& Frangou, 2004; Strakowski et al. 2004; Frey et al. 2007). Behavioural studies have shown some evidence of memory impairment in bipolar disorder (Frey et al. 2007), but it has not been possible to determine whether these results derive from deficits in hippocampal function or from impairments in other brain regions involved in memory encoding and retrieval such as the prefrontal cortex (PFC). Functional neuroimaging studies have shown some evidence of increased activation of the hippocampus in bipolar disorder in affect processing tasks (Lawrence et al. 2004; Chen et al. 2006), but no studies have investigated hippocampal function in bipolar disorder in memory tasks known to depend upon this brain region.

In the present study we compared hippocampal activation in patients with schizophrenia and bipolar disorder and also in unaffected comparison subjects during the learning of novel face-name pair associations. The formation of complex cross-modal associations, such as face-name pairs, is known to depend on the hippocampus (Vargha-Khadem et al. 1997). Functional imaging studies have shown that activation of the anterior hippocampus during facename pair learning correlates with successful memory encoding (Sperling et al. 2003; Zeineh et al. 2003; Kirwan \& Stark, 2004). Here we show that patients with schizophrenia and bipolar disorder show distinct patterns of hippocampal and PFC activation during the encoding and retrieval of face-name pairs despite closely matched behavioural performance. These results suggest a differential dysregulation of frontotemporal neural systems in the two disorders.

\section{Method}

\section{Participants}

Patients meeting DSM-IV diagnostic criteria for schizophrenia or bipolar disorder (type I) were recruited from out-patient and clinically stable inpatient populations in Edinburgh. Diagnoses were confirmed using the Structured Clinical Interview for
DSM-IV (SCID). Individuals meeting diagnostic criteria for schizo-affective disorder were excluded from the study. Further exclusion criteria were age under 18 or over 65 years, neurological disease, current dependence on alcohol or non-prescribed drugs, and other concomitant axis I disorders. Clinical symptoms were assessed on the day of scanning using the Positive and Negative Syndrome Scale (PANSS; Kay et al. 1987), the Hamilton Depression Rating Scale (HAMD; Hamilton, 1960) and the Young Mania Rating Scale (YMRS; Young et al. 1978). Healthy control volunteers were recruited from the same regions and communities as the patients. The diagnostic status of the control subjects was also confirmed by the SCID. Control participants were screened for any family history of psychiatric illness and were also subject to the same exclusion criteria as the patients.

A total of 48 subjects were recruited (16 in each group); however, two subjects were excluded from further analysis because of a failure to make behavioural responses during scanning (one each from the control and bipolar groups) and three subjects were excluded because of scanner and movement artefacts. The final groups consisted of 15 patients with schizophrenia, 14 patients with bipolar disorder and 14 controls.

The study was approved by the local ethics committee, and after complete description of the study, written informed consent was obtained from all participants.

\section{Experimental paradigm}

A repeated face-name pair encoding and retrieval task was used to investigate the acquisition of associative memory over time, based on previous studies (Zeineh et al. 2003). Subjects were required to learn a series of six face-name pairs in each of three task runs. Runs consisted of four blocks of encoding (40 s per block), four blocks of retrieval (40 s per block) and interspersed baseline blocks of fixation (14 s per block). During each of the encoding blocks subjects viewed a 2.5-s instruction screen followed by six face-name pairings in random order with each pair shown for $5 \mathrm{~s}$ and with a 1.25-s break between pairs. The pairs remained the same within a given run, but were changed between runs. During retrieval blocks subject were shown the six faces in random order in addition to all six names and were required to press a one of six buttons (three with each hand) to indicate which name had previously been paired with the face. Timings during the retrieval blocks were the same as for the encoding blocks. During fixation blocks subjects viewed a fixation cross. For functional magnetic resonance imaging (fMRI) analysis, sessions were 
divided into early (blocks 1 and 2) and late (blocks 3 and 4) encoding and retrieval periods based on prior evidence that the hippocampus is recruited preferentially during early encoding (Zeineh et al. 2003). Supplementary analyses confirmed that hippocampal activation did not differ between blocks within the early and late training periods in control subjects or patient groups. The number of successfully retrieved face-name pairings was recorded during each retrieval block, providing a within-scanner measure of behavioural performance.

\section{Scanning procedure}

Imaging was carried out at the Brain Imaging Research Centre (BIRC) for Scotland on a GE 1.5-T Signa scanner (GE Medical, USA). The imaging protocol consisted of a localizer scan, followed by a T2-weighted fast spin-echo sequence, two functional imaging paradigms (only one of which is described here), and finally a structural T1-weighted sequence. For the face-name pair task, axial gradient-echo planar images (EPIs) [repetition time $(\mathrm{TR})=2000 \mathrm{~ms}$, echo time $(\mathrm{TE})=40 \mathrm{~ms}$, matrix $=64 \times 64$, field of view $(\mathrm{FOV})=24 \mathrm{~cm}$ ] were acquired continually over two runs. Twenty-eight contiguous interleaved 5-mm slices aligned to the anterior and posterior commissure were acquired within each TR period. Each acquisition consisted of 227 volumes per run, of which the first four volumes were discarded. The T1 sequence yielded 128 contiguous 1.7-mm coronal slices of $256 \times 192$ voxels [acquisition parameters: $\mathrm{TR}=8.1 \mathrm{~ms}, \mathrm{TE}=$ $3.3 \mathrm{~ms}$, inversion time $(\mathrm{TI})=600 \mathrm{~ms}$, number of excitations $(\mathrm{NEX})=1$, flip angle $=15^{\circ}, \mathrm{FOV}=220 \mathrm{~mm}$ ].

\section{Scan processing and analysis}

EPIs were reconstructed offline into ANALYZE format (Mayo Foundation, USA) using DICOM convert functions available in SPM (Statistical Parametric Mapping; The Wellcome Department of Cognitive Neurology and collaborators, Institute of Neurology, UK) running in Matlab (MathWorks, USA). T1 structural images were reconstructed using MRIcro (University of South Carolina, USA). To assess data quality, reconstructed images were examined using 'Art Repair' software (Centre for Interdisciplinary Brain Sciences Research, Stanford University, USA). Images were corrected for differences in image acquisition time between slices (slice timing) and then realigned to the mean functional image using a two-pass procedure to correct for movement artefact throughout the period of image acquisition. The structural (source) and functional (reference) images were then co-registered and the anatomical image was then segmented, creating grey- and white-matter images. The spatial normalization parameters generated from the previous step were then used to normalize the realigned functional EPI data. Finally, the slice timed, realigned and normalized images were smoothed with a 6-mm full-width half-maximum (FWHM) Gaussian filter to meet assumptions for statistical analysis.

Statistical analysis was performed using the general linear model approach as implemented in SPM5. At the individual participant level, the data were modelled with each of the task conditions (encoding blocks, retrieval blocks and fixation blocks) modelled by a boxcar function convolved with a synthetic haemodynamic response function. Estimates of head movement from the realignment stage of preprocessing were included as additional regressors in the first-level model. Before fitting the model, the participants' data were filtered in the time domain using a high-pass filter (128 s cut-off) and serial correlations were accounted for by using the autoregressive [AR(1)] model. All pre-processing and analyses were conducted using default settings unless stated otherwise. Contrast images for each participant were then constructed representing a subject-specific summary of brain responses to the different conditions for (1) early encoding versus fixation, (2) late encoding versus fixation, (3) early retrieval versus fixation and (4) late retrieval versus fixation.

Contrast images were entered into a second-level random effects analysis to examine areas of activation within each of the three groups (one-sample $t$ test) and differences in activation between the groups (ANOVA) for the main contrasts of interest. Pairwise comparisons between groups were conducted within a design matrix incorporating all three groups. Between-group statistical maps were thresholded at a level of $p=0.001$ uncorrected, and regions were considered significant at the $p<0.05$ cluster level corrected for multiple comparisons across the whole brain volume. Based on our prior hypothesis, small volume corrections (SVCs) were applied for the bilateral hippocampal formation derived from the WFU PickAtlas (Tzourio-Mazoyer et al. 2002; Maldjian et al. 2003).

\section{Correlation analysis}

Correlation analyses were performed to investigate the relationship of the between-group differences to clinical variables. Data were extracted using the SPM VOI function from spheres of $4 \mathrm{~mm}$ radius around the peak voxel from clusters showing between-group differences in the encoding and retrieval sessions. These data were then correlated within each of the patient groups with symptom scores on the PANSS (positive, 
Table 1. Demographic and clinical characteristics

\begin{tabular}{|c|c|c|c|c|c|c|}
\hline & \multicolumn{2}{|c|}{ Schizophrenia group $(n=15)$} & \multicolumn{2}{|c|}{ Bipolar group $(n=14)$} & \multicolumn{2}{|c|}{ Control group $(n=14)$} \\
\hline & Mean & S.D. & Mean & S.D. & Mean & S.D. \\
\hline Age (years) & 38.1 & 10.4 & 40.8 & 10.4 & 33.7 & 7.9 \\
\hline NART IQ & 113.6 & 6.7 & 111.1 & 8.6 & 114.8 & 8.9 \\
\hline PANSS positive & 13.0 & 3.8 & 10.6 & 4.2 & & \\
\hline PANSS negative & 12.3 & 2.8 & 8.2 & 2.7 & & \\
\hline PANSS total & 48.4 & 10.3 & 42.2 & 13.3 & & \\
\hline HAMD & 4.3 & 3.8 & 6.4 & 8.0 & & \\
\hline \multirow[t]{2}{*}{ YMRS } & 1.9 & 2.8 & 2.6 & 3.7 & & \\
\hline & $n$ & $\%$ & $n$ & $\%$ & $n$ & $\%$ \\
\hline Male & 12 & 80 & 10 & 71 & 10 & 71 \\
\hline Right-handed & 9 & 60 & 11 & 79 & 12 & 85 \\
\hline Smokers & 7 & 47 & 6 & 43 & 4 & 28 \\
\hline Antipsychotic & 15 & 100 & 8 & 57 & & \\
\hline Antidepressant & 5 & 33 & 4 & 29 & & \\
\hline Lithium & 0 & 0 & 5 & 36 & & \\
\hline Valproate & 0 & 0 & 3 & 21 & & \\
\hline
\end{tabular}

NART, National Adult Reading Test; PANSS, Positive and Negative Syndrome Scale; HAMD, Hamilton Depression Rating Scale; YMRS, Young Mania Rating Scale; S.D., standard deviation.

negative, general and total), HAMD, YMRS and medication variables. Antipsychotic medication doses were converted into chlorpromazine equivalents (Woods, 2003) for correlation analysis.

\section{Discriminant function analysis}

To assess whether between-group differences in activation separated the patient groups with potentially clinically useful precision, a discriminant function analysis was conducted using the extracted data from the clusters showing between-group differences. We assumed that the groups could be separated by a straight line on a scatter plot of their activation scores; in other words, that the discriminant function was linear. The usefulness of fMRI activations as discriminators between the groups was assessed by comparing a subject's known diagnosis with that predicted from their pattern of fMRI activation (McIntosh et al. 2008).

\section{Results}

\section{Participant characteristics and behaviour}

The demographic and clinical characteristics of the participants are presented in Table 1 and full details of medication doses are shown in Supplementary Table 1 (available online). There were no significant differences between the groups in terms of age, IQ measured by the National Adult Reading Test (NART; Nelson \& Willison, 1991), gender, handedness or number of smokers. Control subjects smoked on average a lower total number of cigarettes per day than the patient groups, but there was no difference between the schizophrenia and bipolar groups in the mean number of cigarettes smoked (10 cigarettes/day on average in the schizophrenia group and 11 cigarettes/day on average in the bipolar group). There were no significant difference between the groups in terms of PANSS positive, PANSS general or PANSS total scores, but patients with schizophrenia had higher PANSS negative syndrome scores than patients with bipolar disorder $(p<0.001)$. Depression and mania rating scores did not differ significantly between the patient groups. Four patients with bipolar disorder and two patients with schizophrenia had HAMD scores $>8$ on the day of scanning. Two patients with bipolar disorder and no patients with schizophrenia had YMRS scores $>8$ on the day of scanning. Twelve out of 14 patients with bipolar disorder had a lifetime history of psychotic symptoms during mood episodes.

The groups were well matched in terms of inscanner behavioural performance (Fig. 1). All subject groups showed learning of the face-name pair association across training, with the majority of new learning occurring during the early encoding sessions. 


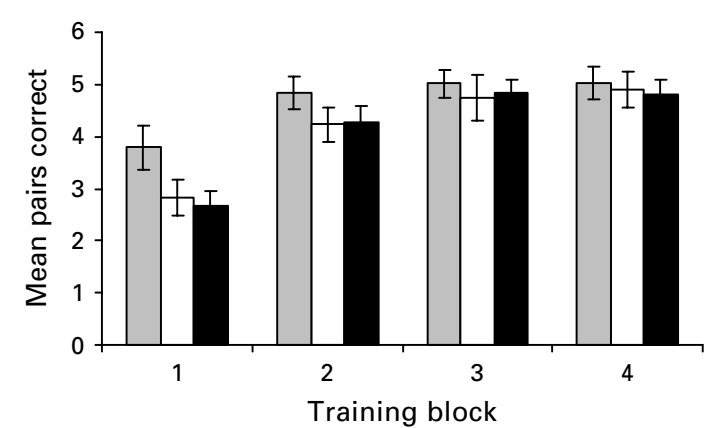

Fig. 1. Number of face-name pairs correctly remembered at each retrieval block by group. Error bars show standard error of the mean. $\square$, Control subjects ; $\square$, bipolar disorder subjects; $\mathbf{\square}$, schizophrenia subjects.

Repeated-measures ANOVA confirmed a main effect of session $(F=60, \mathrm{df}=3,120, p<0.001)$ but no main effect of group $(F=1.0, \mathrm{df}=2,40, p=0.4)$ or group by session interaction $(F=1.5, \mathrm{df}=6,120, p=0.2)$. Notably, the schizophrenia and bipolar groups had almost identical performance across all training sessions.

\section{Task-related brain activation}

A full list of areas activated by the task in control subjects is shown in Supplementary Table 2 (available online). Activation was seen in the anterior hippocampus during early encoding but not during late encoding. This pattern of results is highly consistent with previous studies of face-name pair learning (Zeineh et al. 2003; Kirwan \& Stark, 2004) and with more general evidence supporting a selective role for the anterior hippocampus in encoding (Lepage et al. 1998; Dolan \& Fletcher, 1999). Pronounced activation of the posterior, but not the anterior, hippocampus was seen during both retrieval periods. Activation of frontal cortical areas, including dorsal, inferior and superior frontal cortices, was also seen during both encoding and retrieval (Supplementary Table 2). Full details of the within-group activation patterns for the patient groups are shown in Supplementary Tables 3 and 4 (available online).

\section{Between-group comparisons}

Regions showing significant between-group differences during the encoding and retrieval of face-name pairs are presented in Table 2.

During early encoding, patients with schizophrenia showed significantly less relative activation of the anterior right hippocampus than patients with bipolar disorder [corrected $p=0.034$ within bilateral hippocampal SVC, $z=4.07$, total cluster size $=64$ voxels, peak Montreal Neurological Institute (MNI) coordinates $34,-6,-24$ (Fig. 2)]. No other brain regions showed this pattern of activation differences between the groups. Inspection of the parameter estimates for this cluster revealed that this effect derived from lower hippocampal activation in the schizophrenia group than in the bipolar and control groups (Fig. 2). By contrast, bipolar subjects showed significantly lower activation of the left dorsolateral prefrontal cortex (DLPFC) during early encoding relative to subjects with schizophrenia [Brodmann area (BA) 8, corrected $p=0.002, z=3.80$, cluster $=307$ voxels, peak MNI coordinates $-16,38,48$ (Fig. 2)]. Inspection of the parameter estimates confirmed that bipolar subjects had lower activation of this brain region than either patients with schizophrenia or control subjects (Fig. 2). Unaffected comparison subjects showed greater activation of the midline cerebellum than patients with schizophrenia during early encoding (corrected $p=$ $0.001, z=4.47$, cluster $=372$ voxels, peak MNI coordinates $4,-42,-12$ ).

During early retrieval, patients with schizophrenia showed greater activation than bipolar subjects of the dorsomedial prefrontal cortex (DMPFC), extending to the dorsal cingulate cortex [BA 8, corrected $p=0.021, z=4.20$, cluster $=276$ voxels, peak MNI coordinates $-8,22,42$ (Fig. 2)]. Patients with schizophrenia also showed greater activation of the dorsal PFC than control subjects during this task phase across a larger cluster that extended to include the region discriminating the patient groups and also to an additional area of DLPFC (BA 6/8, corrected $p=0.006$, $z=4.20$, cluster $=373$ voxels, peak MNI coordinates $-32,16,54)$.

There were no clusters showing discrimination between the patient groups during late encoding. Control subjects showed greater activation of the left superior temporal cortex than patients with schizophrenia in late encoding (corrected $p=0.004$, $z=4.37$, cluster $=276$ voxels, peak MNI coordinates $-38,-10,0)$ whereas patients with schizophrenia showed greater activation of the lingual gyrus than controls during this task period (corrected $p=0.024$, $z=4.18$, cluster $=189$ voxels, peak MNI coordinates $-12,-82,-6)$.

During late retrieval, patients with schizophrenia continued to show greater activation of the DMPFC than patients with bipolar disorder (BA 8, corrected $p=0.009, z=4.68$, cluster $=314$ voxels, peak MNI coordinates $-6,24,40)$.

\section{Symptom and medication correlations}

We investigated whether the differences between the schizophrenia and bipolar groups related to mood state, psychotic symptoms or medication dose. Data were extracted from brain regions showing group 
Table 2. Between-group activation differences

\begin{tabular}{llllll}
\hline & $p_{\text {corr }}$ & $K_{\mathrm{E}}$ & Peak $z$ & Peak coordinates & Region \\
\hline 1. Early encoding & & & & & \\
$\quad$ Bipolar $>$ Schizophrenia & $0.034^{\mathrm{a}}$ & 64 & 4.07 & $34,-6,-24$ & Right hippocampus \\
$\quad$ Schizophrenia $>$ Bipolar & 0.002 & 307 & 3.80 & $-16,38,48$ & Left DLPFC (BA 8) \\
$\quad$ Control $>$ Schizophrenia & 0.001 & 372 & 4.47 & $4,-42,-12$ & Midline cerebellum \\
$\begin{array}{l}\text { 2. Early retrieval } \\
\quad \text { Schizophrenia }>\text { Bipolar }\end{array}$ & 0.021 & 276 & 4.20 & $-8,22,42$ & Left DMPFC (BA 8) \\
$\quad$ Schizophrenia $>$ Control & 0.006 & 373 & 4.20 & $-32,16,54$ & Left DLPFC (BA 6/8) \\
$\begin{array}{l}\text { 3. Late encoding } \\
\quad \text { Control }>\text { Schizophrenia }\end{array}$ & 0.004 & 276 & 4.37 & $-38,-10,0$ & Left superior temporal cortex \\
$\quad$ Schizophrenia $>$ Control & 0.024 & 189 & 4.18 & $-12,-82,-6$ & Left lingual gyrus \\
4. Late retrieval & & & & & Left DMPFC (BA 8) \\
$\quad$ Schizophrenia $>$ Bipolar & 0.009 & 314 & 4.68 & $-6,24,40$ & \\
\hline
\end{tabular}

DLPFC, Dorsolateral prefrontal cortex; DMPFC, dorsomedial prefrontal cortex; BA, Brodmann area.

${ }^{a}$ Within a hippocampal small volume correction (SVC). Only contrasts with significant between-group differences are shown.

differences and correlated with symptom scores (HAMD, YMRS, PANNS positive, PANSS negative, PANSS general and PANSS total) and medication dose (chlorpromazine equivalents, lithium dose, valproate dose) within each group. There was a positive correlation between YMRS score and hippocampal activation during early encoding in the bipolar group $(p=0.01)$ and a negative correlation between YMRS and hippocampal activation during early encoding in the schizophrenia group $(p<0.05)$. The latter effect was accounted for by patients with schizophrenia scoring on the items in the YMRS rating abnormal thought content and lack of insight (correlation $p<0.05$ for these items only). No significant correlations were found with any of the other symptom measures. There was no evidence of any effect of medication on brain activation in the regions of interest except for a positive correlation between antipsychotic dose and dorsal prefrontal activation during late retrieval in the bipolar group $(p<0.05)$. Notably, this effect of medication operated to reduce, rather than increase, the difference between patient groups. Comparison of bipolar subjects with $(n=8)$ and without $(n=6)$ mood stabilizer medication also did not reveal any differences in blood oxygen leveldependent (BOLD) signal due to medication.

\section{Discriminant function analysis}

Data were extracted from the four clusters showing differences in activation between the schizophrenia and bipolar groups and entered into a discriminant function analysis. The model that best differentiated the patient groups included the hippocampal and dorsal prefrontal clusters from the early encoding period and the dorsal prefrontal cluster from the early retrieval period. This model classified $96 \%$ of patients correctly (Fig. 3). Notably, this analysis was performed only to explore the degree to which these activation differences discriminated the groups within the current study, and further prospective investigation would be required to investigate the predictive and diagnostic value of these findings.

\section{Discussion}

Very few studies have compared the neural basis of schizophrenia and bipolar disorder using functional neuroimaging (Curtis et al. 2001; McIntosh et al. 2008). The few studies that have made such a direct comparison have focused on tests of frontal lobe function (Curtis et al. 2001; McIntosh et al. 2008). The degree to which abnormalities in brain function in other regions, such as the temporal lobes, separate the two disorders therefore remains largely unclear. Previous evidence from studies focusing on each disorder separately suggests that they may differ in terms of hippocampal function. We therefore investigated whether hippocampal activation could distinguish schizophrenia from bipolar disorder using fMRI in participants performing an associative memory task known to depend on this brain region. Our results demonstrate differential activation of the hippocampus in the two disorders, which was evident despite closely matched behavioural performance measured during scanning.

Patients with schizophrenia showed decreased anterior hippocampal activation during memory 
(a)
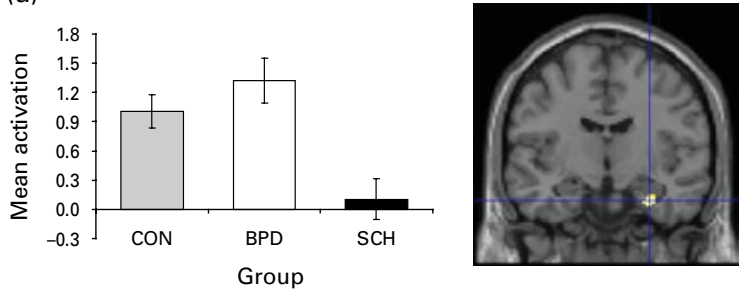

(b)
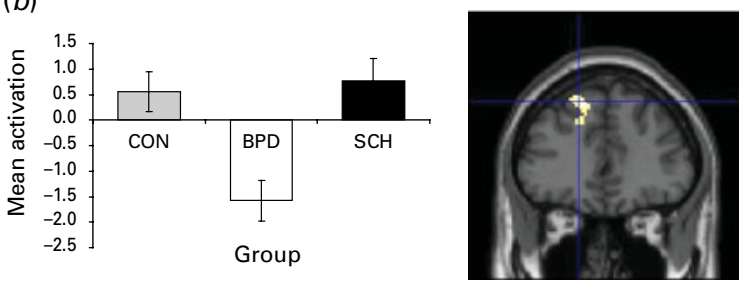

(c)

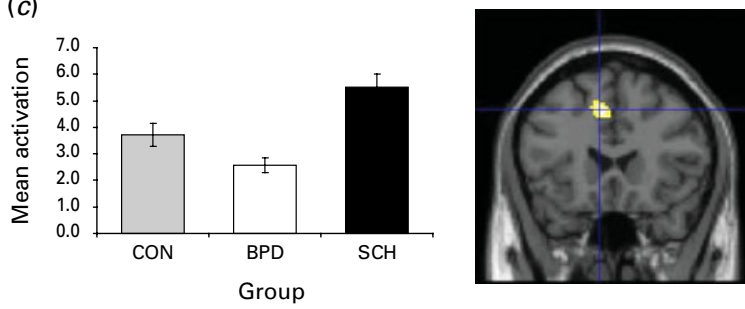

Fig. 2. Clusters showing discrimination between the patient groups. (a) Decreased anterior hippocampal activation during early encoding in patients with schizophrenia compared to patients with bipolar disorder. (b) Decreased dorsolateral prefrontal cortex (DLPFC) activation during early encoding in patients with bipolar disorder compared to patients with schizophrenia. (c) Decreased dorsomedial prefrontal cortex (DMPFC) activation in subjects with bipolar disorder compared to subjects with schizophrenia during early retrieval. SPMs thresholded at $p=0.001$. Blue cross-hairs show voxel of peak difference. Error bars show standard error of the mean. CON, control subjects; BPD, bipolar disorder subjects; $\mathrm{SCH}$, schizophrenia subjects.

encoding, which distinguished them from subjects with bipolar disorder. Examination of parameter estimates demonstrated lower hippocampal activation in the schizophrenia group than in either the control group or the bipolar disorder group, although the difference between the schizophrenia group and the control subjects failed to reach cluster-level significance (Fig. 2). The anterior hippocampal formation is known to play a central role in the memory encoding, and specifically in the encoding of cross-domain associations such as face-name pairs (Lepage et al. 1998; Zeineh et al. 2003; Kirwan \& Stark, 2004). The formation of integrative, cross-domain associations is thought to be central to the ability to encode and recall episodic memories (Mayes et al. 2007). Patients with schizophrenia have memory impairments that are

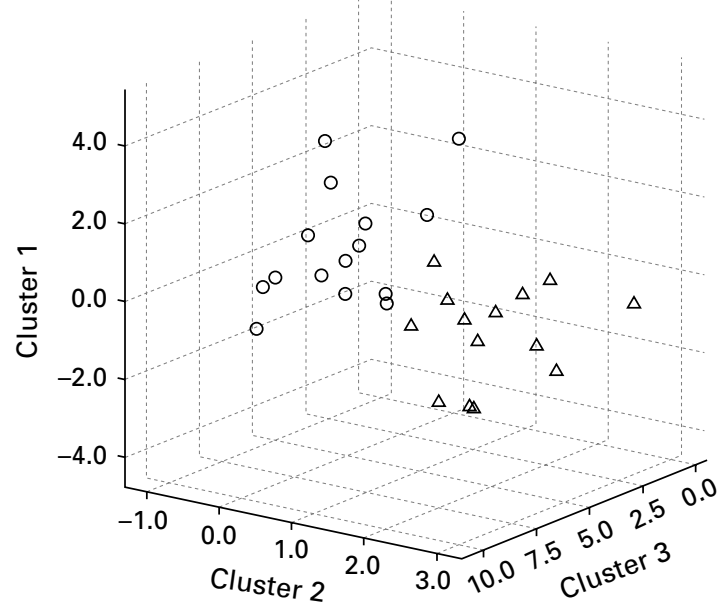

Fig. 3. Three-dimensional scatter plot showing separation of the schizophrenia and bipolar groups based on functional magnetic resonance imaging (fMRI) measures. Cluster 1, anterior hippocampal cluster, early encoding ; cluster 2, dorsolateral prefrontal cluster, early encoding; cluster 3, dorsomedial prefrontal cluster, early retrieval; circles, schizophrenia subjects; triangles, bipolar subjects.

particularly marked for tasks that depend on the hippocampus such as tests of associative and episodic memory (Huron et al. 1995; Danion et al. 1999; Achim \& Lepage, 2003; Toulopoulou et al. 2003). Similar deficits in memory function are also seen in the relatives of patients with schizophrenia, suggesting that they represent a heritable vulnerability for the disorder (Toulopoulou et al. 2003). Functional imaging studies have demonstrated impairments in hippocampal activation in schizophrenia that are particularly prominent in memory tasks (Heckers et al. 1998; Heckers, 2001; Achim \& Lepage, 2005; Ongur et al. 2006). This failure of activation may derive in part from tonic hyperactivation of this brain region, which has been associated with abnormal regulation of ascending dopaminergic function in models of schizophrenia (Lodge \& Grace, 2007; Lisman et al. 2008). By contrast, there is little consistent evidence of either structural or functional abnormalities in the hippocampus in bipolar disorder, other than increased activation in response to emotional stimuli, which may relate to the close functional integration of the hippocampus with the adjacent amygdala (Haldane \& Frangou, 2004; Strakowski et al. 2004; Frey et al. 2007). In the present study we found a positive correlation between mania symptoms and hippocampal activation in the bipolar group, which may similarly reflect an influence of affective state on hippocampal activation in bipolar subjects.

Subjects with bipolar disorder showed decreased activation of the DLPFC during the encoding of 
face-name pairs compared to patients with schizophrenia, who did not differ significantly from control subjects in activation of this region during encoding (Fig. 2). Frontal lobe regions, including the DLPFC, play a central role in organizational processes related to memory encoding (Blumenfeld \& Ranganath, 2007) and have been shown to be particularly important for the encoding of associative memories (Summerfield et al. 2006). There is evidence of impaired function of both dorsal and ventral prefrontal cortical regions in bipolar disorder, which may account for both the neuropsychological deficits and dysregulation of emotional control seen in patients with this disorder (Blumberg et al. 2003; Strakowski et al. 2004; Phillips \& Vieta, 2007). Decreased dorsal PFC activation has previously been reported in euthymic bipolar patients during a verbal encoding task (Deckersbach et al. 2006) and in fMRI studies of working memory (Monks et al. 2004; Lagopoulos et al. 2007). The present results support the view that dorsal PFC activation is abnormal during memory encoding in bipolar disorder, a finding that may reflect more general impairments in executive control in the disorder (Phillips \& Vieta, 2007). Impairments in frontal lobe function are therefore more likely to underlie the reported deficits in memory function in bipolar disorder than deficits in hippocampal function.

Participants with schizophrenia showed increased activation of left DMPFC during recall periods compared to patients with bipolar disorder and increased activation of the DMPFC and the DLPFC during retrieval relative to control participants. The PFC is strongly implicated in memory retrieval, both for the adoption and maintenance of retrieval strategies and for the monitoring of retrieval success (Fletcher et al. 1997). Left dorsal PFC activation has been shown to be greater for items that are consciously recalled rather than being simply familiar (Henson et al. 1999) and to correlate with successful recall of face-name pair memories (Zeineh et al. 2003). Increased activation of the DMPFC and additional recruitment of DLPFC in patients with schizophrenia may reflect the higher demand placed on this cortical region in schizophrenia subjects to maintain task performance. This increase in demand could reflect either the relatively inefficient prefrontal activation in schizophrenia or a compensation for impaired hippocampal activation (Heckers et al. 1998; Callicott et al. 2003).

The current study is the first to directly compare brain activations in schizophrenia and bipolar disorder using a memory task designed to recruit the hippocampus. A strength of the study was the balanced task performance across subject groups, which meant that the between-group differences in brain activation could not be accounted for by differences in performance. Future studies, however, could also use event-related fMRI and subsequent memory analysis to isolate only those events that were successfully remembered by a given subject. A limitation of the current study is that all the patients were, of necessity, on medication. There was, however, substantial overlap in medication between the patient groups, and medication effects did not explain the observed group differences in hippocampal and prefrontal activation, with the only significant effect of medication tending to decrease, rather than increase, between-group differences in the PFC. In addition, the current study had relatively small group numbers, and studies with larger sample sizes would be required to confirm the reliability and replicability of the current findings. Although discriminant function analysis suggested that this task produced a significant separation between the schizophrenia and bipolar groups, this represented a post-hoc investigation of the data, which would require confirmation in an independent sample. Furthermore, it should be noted that the discriminant function analysis compared the two patient groups, and did not examine the separation of patients from controls.

Overall, the present results show that schizophrenia and bipolar disorder can be distinguished in terms of activation of the hippocampus and PFC during memory encoding and retrieval. The ability to separate the two disorders using non-invasive imaging thus supports the view that there are separable components to the pathology underlying the two disorders and is of direct relevance to efforts to construct valid classifications of psychiatric illness.

\section{Acknowledgements}

J.H. was supported by a Medical Research Council (MRC) Clinical Research Training Fellowship. A.M. was supported by the Health Foundation. The work was supported by an award (EU_036) from the Translational Medicine Research Collaboration, a consortium made up of the Universities of Aberdeen, Dundee, Edinburgh and Glasgow, the four associated National Health Service (NHS) Health Boards (Grampian, Tayside, Lothian, and Greater Glasgow and Clyde), Scottish Enterprise, and Wyeth Pharmaceuticals. The study was carried out at the SFC Brain Imaging Research Centre (www.sbirc.ed.ac.uk) and the Wellcome Trust Clinical Research Facility, University of Edinburgh, Western General Hospital, Edinburgh. The work was carried out on a 1.5-T Signa Horizon MR/I 1.5T HDX operating under a research collaboration with GE Medical Systems (USA), operating as IGE in the UK. 


\section{Declaration of Interest}

J.H., A.McI., H.C.W. and S.M.L. have received grants from the Translational Medicine Research Collaboration, a consortium made up of the Universities of Aberdeen, Dundee, Edinburgh and Glasgow, the four associated NHS Health Boards (Grampian, Tayside, Lothian, and Greater Glasgow and Clyde), Scottish Enterprise, and Wyeth Pharmaceuticals. H.I.W. is an employee of Wyeth Pharmaceuticals.

\section{Note}

Supplementary material accompanies this paper on the Journal's website (http:/ /journals.cambridge.org/ psm).

\section{References}

Achim AM, Lepage M (2003). Is associative recognition more impaired than item recognition memory in schizophrenia? A meta-analysis. Brain and Cognition 53, 121-124.

Achim AM, Lepage M (2005). Episodic memory-related activation in schizophrenia : meta-analysis. British Journal of Psychiatry 187, 500-509.

Aleman A, Hijman R, de Haan EHF, Kahn RS (1999). Memory impairment in schizophrenia : a meta-analysis. American Journal of Psychiatry 156, 1358-1366.

Blumberg HP, Leung H-C, Skudlarski P, Lacadie CM, Fredericks CA, Harris BC, Charney DS, Gore JC, Krystal JH, Peterson BS (2003). A functional magnetic resonance imaging study of bipolar disorder: state- and trait-related dysfunction in ventral prefrontal cortices. Archives of General Psychiatry 60, 601-609.

Blumenfeld RS, Ranganath C (2007). Prefrontal cortex and long-term memory encoding: an integrative review of findings from neuropsychology and neuroimaging. Neuroscientist 13, 280-291.

Boos HBM, Aleman A, Cahn W, Pol HH, Kahn RS (2007). Brain volumes in relatives of patients with schizophrenia: a meta-analysis. Archives of General Psychiatry 64, 297-304.

Callicott JH, Mattay VS, Verchinski BA, Marenco S, Egan MF, Weinberger DR (2003). Complexity of prefrontal cortical dysfunction in schizophrenia: more than up or down. American Journal of Psychiatry 160, 2209-2215.

Chen C-H, Lennox B, Jacob R, Calder A, Lupson V, Bisbrown-Chippendale R, Suckling J, Bullmore E (2006). Explicit and implicit facial affect recognition in manic and depressed states of bipolar disorder: a functional magnetic resonance imaging study. Biological Psychiatry 59, 31-39.

Curtis VA, Dixon TA, Morris RG, Bullmore ET, Brammer MJ, Williams SCR, Sharma T, Murray RM, McGuire PK (2001). Differential frontal activation in schizophrenia and bipolar illness during verbal fluency. Journal of Affective Disorders 66, 111-121.

Danion J-M, Rizzo L, Bruant A (1999). Functional mechanisms underlying impaired recognition memory and conscious awareness in patients with schizophrenia. Archives of General Psychiatry 56, 639-644.
Deckersbach T, Dougherty DD, Savage C, McMurrich S, Fischman AJ, Nierenberg A, Sachs G, Rauch SL (2006). Impaired recruitment of the dorsolateral prefrontal cortex and hippocampus during encoding in bipolar disorder. Biological Psychiatry 59, 138-146.

Dolan RJ, Fletcher PF (1999). Encoding and retrieval in human medial temporal lobes: an empirical investigation using functional magnetic resonance imaging (fMRI). Hippocampus 9, 25-34.

Fletcher PC, Frith CD, Rugg MD (1997). The functional neuroanatomy of episodic memory. Trends in Neurosciences 20, 213-218.

Frey BN, Andreazza AC, Nery FG, Martins MR, Quevedo J, Soares JC, Kapczinski F (2007). The role of hippocampus in the pathophysiology of bipolar disorder. Behavioural Pharmacology 18, 419-430.

Haldane M, Frangou S (2004). New insights help define the pathophysiology of bipolar affective disorder: neuroimaging and neuropathology findings. Progress in Neuro-Psychopharmacology and Biological Psychiatry 28, 943-960.

Hamilton M (1960). A rating scale for depression. Journal of Neurology, Neurosurgery and Psychiatry 23, 56-62.

Heckers S (2001). Neuroimaging studies of the hippocampus in schizophrenia. Hippocampus 11, 520-528.

Heckers S, Rauch S, Goff D, Savage C, Schacter D, Fischman A, Alpert N (1998). Impaired recruitment of the hippocampus during conscious recollection in schizophrenia. Nature Neuroscience 1, 318-323.

Henson RNA, Rugg MD, Shallice T, Josephs O, Dolan RJ (1999). Recollection and familiarity in recognition memory: an event-related functional magnetic resonance imaging study. Journal of Neuroscience 19, 3962-3972.

Huron C, Danion JM, Giacomoni F, Grange D, Robert P, Rizzo L (1995). Impairment of recognition memory with, but not without, conscious recollection in schizophrenia. American Journal of Psychiatry 152, 1737-1742.

Kay SR, Fiszbein A, Opler LA (1987). The positive and negative syndrome scale (PANSS) for schizophrenia. Schizophrenia Bulletin 13, 261-276.

Kirwan CB, Stark CE (2004). Medial temporal lobe activation during encoding and retrieval of novel face-name pairs. Hippocampus 14, 919-930.

Lagopoulos J, Ivanovski B, Malhi GS (2007). An eventrelated functional MRI study of working memory in euthymic bipolar disorder. Journal of Psychiatry and Neuroscience 32, 174-184.

Lawrence NS, Williams AM, Surguladze S, Giampietro V, Brammer MJ, Andrew C, Frangou S, Ecker C, Phillips ML (2004). Subcortical and ventral prefrontal cortical neural responses to facial expressions distinguish patients with bipolar disorder and major depression. Biological Psychiatry 55, 578-587.

Lepage M, Habib R, Tulving E (1998). Hippocampal PET activations of memory encoding and retrieval: the HIPER model. Hippocampus 8, 313-322.

Lisman JE, Coyle JT, Green RW, Javitt DC, Benes FM, Heckers S, Grace AA (2008). Circuit-based framework for understanding neurotransmitter and risk gene interactions in schizophrenia. Trends in Neurosciences 31, 234-242. 
Lodge DJ, Grace AA (2007). Aberrant hippocampal activity underlies the dopamine dysregulation in an animal model of schizophrenia. Journal of Neuroscience 27, 11424-11430.

Maldjian JA, Laurienti PJ, Kraft RA, Burdette JH (2003).

An automated method for neuroanatomic and cytoarchitectonic atlas-based interrogation of fMRI data sets. NeuroImage 19, 1233-1239.

Mayes A, Montaldi D, Migo E (2007). Associative memory and the medial temporal lobes. Trends in Cognitive Sciences 11, 126-135.

McIntosh AM, Whalley HC, McKirdy J, Hall J, Sussmann JED, Shankar P, Johnstone EC, Lawrie SM (2008). Prefrontal function and activation in bipolar disorder and schizophrenia. American Journal of Psychiatry 165, 378-384.

Monks PJ, Thompson JM, Bullmore ET, Suckling J, Brammer MJ, Williams SC, Simmons A, Giles N, Lloyd AJ, Harrison CL, Seal M, Murray RM, Ferrier IN, Young AH, Curtis VA (2004). A functional MRI study of working memory task in euthymic bipolar disorder: evidence for task-specific dysfunction. Bipolar Disorders 6 , 550-564.

Nelson HE, Willison JR (1991). The Revised National Adult Reading Test - Test Manual. NFER-Nelson: Windsor, UK.

Ongur D, Cullen TJ, Wolf DH, Rohan M, Barreira P, Zalesak M, Heckers S (2006). The neural basis of relational memory deficits in schizophrenia. Archives of General Psychiatry 63, 356-365.

Phillips ML, Vieta E (2007). Identifying functional neuroimaging biomarkers of bipolar disorder: toward DSM-V. Schizophrenia Bulletin 33, 893-904.

Sperling R, Chua E, Cocchiarella A, Rand-Giovannetti E, Poldrack R, Schacter DL, Albert M (2003). Putting names to faces: successful encoding of associative memories activates the anterior hippocampal formation. NeuroImage 20, 1400-1410.

Strakowski SM, DelBello MP, Adler CM (2004). The functional neuroanatomy of bipolar disorder: a review of neuroimaging findings. Molecular Psychiatry 10, 105-116.

Summerfield C, Greene M, Wager T, Egner T, Hirsch J, Mangels J (2006). Neocortical connectivity during episodic memory formation. PLoS Biology 4, e128.

Toulopoulou T, Rabe-Hesketh S, King H, Murray RM, Morris RG (2003). Episodic memory in schizophrenic patients and their relatives. Schizophrenia Research 63, 261-271.

Tzourio-Mazoyer N, Landeau B, Papathanassiou D, Crivello F, Etard O, Delcroix N, Mazoyer B, Joliot M (2002). Automated anatomical labeling of activations in SPM using a macroscopic anatomical parcellation of the MNI MRI single-subject brain. NeuroImage 15, 273-289.

Vargha-Khadem F, Gadian DG, Watkins KE, Connelly A, Van Paesschen W, Mishkin M (1997). Differential effects of early hippocampal pathology on episodic and semantic memory. Science 277, 376-380.

Woods SW (2003). Chlorpromazine equivalent doses of the newer atypical antipsychotics. Journal of Clinical Psychiatry 64, 663-667.

Wright IC, Rabe-Hesketh S, Woodruff PWR, David AS, Murray RM, Bullmore ET (2000). Meta-analysis of regional brain volumes in schizophrenia. American Journal of Psychiatry 157, 16-25.

Young RC, Biggs JT, Ziegler VE, Meyer DA (1978). A rating scale for mania: reliability, validity and sensitivity. British Journal of Psychiatry 133, 429-435.

Zeineh MM, Engel SA, Thompson PM, Bookheimer SY (2003). Dynamics of the hippocampus during encoding and retrieval of face-name pairs. Science 299, 577-580. 